ИЗВЕСТИЯ АКАДЕМИИ НАУК ЭСТОНСКОИ ССР. ТОМ 27 ФИЗИКА * МАТЕМАТИКА, 1978, № 4

\title{
ПРОЦЕССЫ УПРАВЛЕНИЯ В ИНДУКТИВНО-КЛЮЧЕВОЙ ЦЕПИ ФОРМИРОВАНИЯ МНОГОСТУПЕНЧАТОГО РЕГУЛИРУЕМОГО ПЕРЕМЕННОГО ТОКА
}

\author{
(Представлена Н. Эпиком)
}

Эффективным способом улучшения качества выходного переменного напряжения ключевых преобразователей является формирование многоступенчатого выходного тока, по форме близкого к синусоидальному $\left[{ }^{1,2}\right]$. В непрерывных регуляторах переменного напряжения роль формирователя многоступенчатого тока играет индуктивный накопитель энергии $\left[{ }^{3}\right]$, управляемый как полупроводниковыми, так и магнитными ключами в виде однообмоточных насыщающихся дросселей [ $\left.{ }^{4}\right]$, переключающими число витков накопителя. Но вопросы управления этими ключами до сих пор не рассматривались.

В данной статье анализируются электромагнитные процессы в индуктивно-ключевом формирователе многоступенчатого тока, построенном на базе линейного дросселя и магнитных ключей. В частности, устанавливаются закономерности коммутации ключей, которые могут применяться непосредственно в силовых цепях, а также в цепях управления тиристорами, переключающими число витков линейного дросселя.

В качестве примера рассматривается работа схемы индуктивноключевого формирователя трехступенчатого тока (рис. 1), управляемые ключи которого $\mathrm{K} 1-\mathrm{K} 4$ предназначены для коммутирования напряжения $u_{34}=u-u_{\mathrm{H}}$ в моменты $\omega t=\alpha_{4}+n \pi$, где $n=0,1,2, \ldots$ и $\alpha_{1}$ - угол управления мостового регулятора. Управляемые ключи K1-K4 могут быть реализованы как полупроводниковыми, так и магнитными ключами. При этом напряжение на диагонали мостового регулятора имеет вид

$$
u_{12}=\operatorname{sign}\left[\sin \left(\omega t-\alpha_{1}\right)\right]\left(U_{m} \sin \omega t-u_{\mathrm{H}}\right) .
$$

Формирование трехступенчатого тока осуществляется магнитными ключами M1-M3 и секционированным линейным дросселем Др.

Анализ схемы проводится при следующих основных предпосылках: рассеяние всех дросселей регулятора пренебрежимо мало; индуктивность линейного дросселя равна бесконечности; сердечники магнитных ключей $M 1-$ M3 имеют идеальную кривую намагничивания; нагрузка регулятора $R_{\text {н }}$ активная.

В зависимости от угла управления $\alpha_{1}$ в работе мостового регулятора можно выделить три диапазона $\left[{ }^{4}\right]$ : 

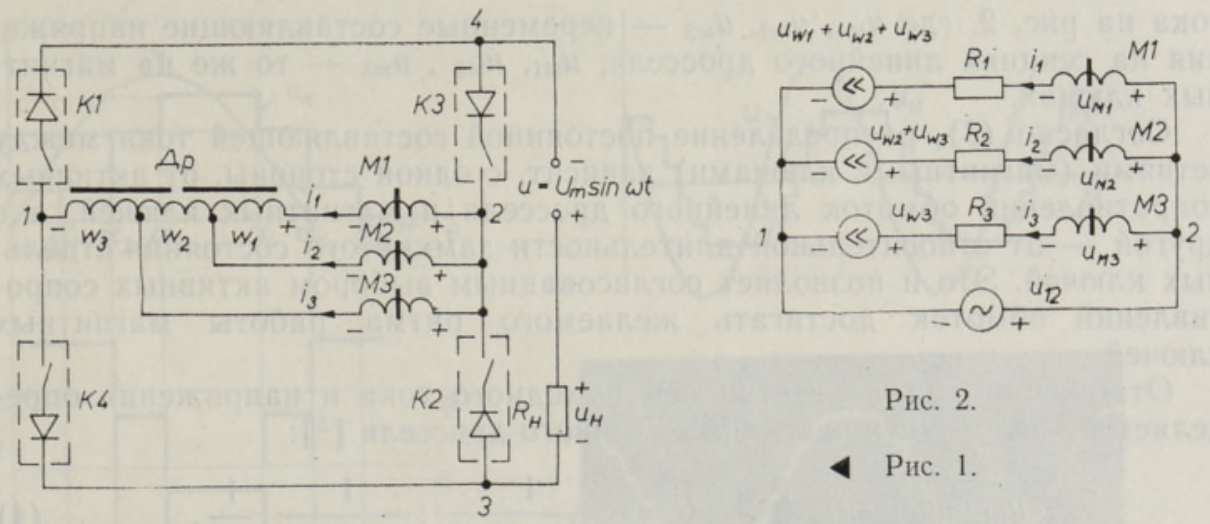

Рис. 2.

4 Рис. 1.

1. $\alpha_{\text {пI }} \leqslant \alpha_{1} \leqslant \pi / 2$, где $\alpha_{\text {п }}$ - предельное значение угла управления. Выходное напряжение $u_{\mathrm{H}}$ имеет ступенчатую форму, т. е. из трех магнитных ключей замкнут только один.

2. $-\pi / 6 \leqslant \alpha_{1} \leqslant \alpha_{\text {п. }}$ В этом случае в выходном напряжении есть интервалы, когда $u_{\mathrm{H}}=u$, т. е. одновременно замкнуты два магнитных ключа (сердечники находятся в насыщенном состоянии).

3. $-\pi / 2 \leqslant \alpha_{1} \leqslant-\pi / 6$. Форма выходного напряжения $u_{\text {н }}$ полностью совпадает с синусоидой.

Проанализируем работу регулятора в первом, основном, диапазоне регулирования.

Так как в параллельных ветвях распределение постоянной составляющей тока обратно пропорционально сопротивлению этих ветвей, то действительны соотношения

$$
\begin{gathered}
I_{01}: I_{02}=\frac{1}{R_{w 1}+R_{\mathrm{M} 1}}: \frac{1}{R_{\mathrm{M} 2}}, \\
\left(I_{01}+I_{02}\right): I_{03}=\frac{1}{R_{w 2}+\frac{R_{\mathrm{M} 2}\left(R_{w 1}+R_{\mathrm{M} 1}\right)}{R_{w 1}+R_{\mathrm{M} 1}+R_{\mathrm{M} 2}}}: \frac{1}{R_{\mathrm{M} 3}},
\end{gathered}
$$

где $I_{0 j}=\frac{1}{\pi} \int_{\alpha_{1}}^{\alpha_{1}+\pi} i_{j} d \Theta, \quad j=1,2,3 ; \Theta=\omega t ; R_{\mathrm{M} 1}, R_{\mathrm{M} 2}, R_{\mathrm{M} 3}-$ активные сопротивления обмоток магнитных ключей; $R_{w 1}, R_{w 2}, R_{w 3}$ - то же обмоток секций линейного дросселя Др.

Преобразуя параллельно-последовательную цепь индуктивно-ключевого формирователя в параллельную, из соотношений (2) получим

$$
I_{01}: I_{02}: I_{03}=\frac{1}{R_{1}}: \frac{1}{R_{2}}: \frac{1}{R_{3}},
$$

где $R_{1}=R_{w 1}+R_{\mathrm{M} 1}, \quad R_{2}=R_{\mathrm{M} 2}, \quad R_{3}=\frac{\left(R_{w 1}+R_{\mathrm{M} 1}\right) R_{\mathrm{M} 2} R_{\mathrm{M} 3}}{R_{w 2}\left(R_{w 1}+R_{\mathrm{M} 1}+R_{\mathrm{M} 2}\right)+R_{\mathrm{M} 2}\left(R_{w 1}+R_{\mathrm{M} 1}\right)}$.

В дальнейшем анализе пренебрегаем активным сопротивлением $R_{w 3}$ секции $w_{3}$ обмотки линейного дросселя, поскольку оно не влияет на распределение тока между магнитными ключами и $\hat{R}_{w 3} \ll R_{\text {н. }}$.

B результате проведенных выше преобразований получим схему замещения индуктивно-ключевой цепи формирования трехступенчатого 
тока на рис. 2 , где $u_{w 1}, u_{w 2}, u_{w 3}$ - переменные составляющие напряжения на секциях линейного дросселя, $u_{\mathrm{M} 1}, u_{\mathrm{M} 2}, u_{\mathrm{M} 3}$ - то же на магнитных ключах.

Согласно (3), распределение постоянной составляющей тока между ветвями (магнитными ключами) зависит, с одной стороны, от активных сопротивлений обмоток линейного дросселя и магнитных ключей, а с другой - от относительной длительности замкнутого состояния отдельных ключей. Это и позволяет согласованным выбором активных сопротивлений обмоток достигать желаемого ритма работы магнитных ключей.

Отношение амплитуд ступеней выходного тока и напряжения определяется числом витков секций линейного дросселя $\left[{ }^{3}\right]$ :

$$
u_{\mathrm{H} 1}: u_{\mathrm{H} 2}: u_{\mathrm{H} 3}=i_{1}: i_{2}: i_{3}=\frac{1}{w_{1}+w_{2}+w_{3}}: \frac{1}{w_{2}+w_{3}}: \frac{1}{w_{3}},
$$

где $u_{\mathrm{H} j}=i_{j} R_{\mathrm{H}}$. Учитывая, что в реальных регуляторах

$$
R_{j} \int_{\alpha_{1}}^{\alpha_{1}+\pi} i_{j} d \Theta \ll \int_{\alpha_{1}}^{\alpha_{1}+\pi} u_{\mathrm{H}} d \Theta
$$

напряжение $u_{w}$ на включенных секциях линейного дросселя в интервале $\alpha_{1} \ll \Theta \ll \alpha_{1}+\pi$ можно принять равным $u_{w}=u_{12}=u-u_{\mathrm{H}}$.

Рассматриваемый нами основной диапазон регулирования $\alpha_{\Pi} \leqslant \alpha_{1} \leqslant$ $\leqslant \pi / 2$ характеризуется тем, что из трех магнитных ключей замкнут только один. Поэтому в интервале $\alpha_{1} \leqslant \Theta \leqslant \alpha_{1}+\pi$, соответствующем полупериоду выходного напряжения, магнитные ключи схемы на рис. 1 и 2 могут находиться в трех различных состояниях: 1) M1 замкнут, M2 и M3 разомкнуты; 2) M2 замкнут, M1 и M3 - разомкнуты; 3) M3 замкнут, M1 и $\mathrm{M} 3$ - разомкнуты.

\begin{tabular}{r|c|c|c}
\multicolumn{3}{c}{ Напряжения на магннтных ключах $u_{\mathrm{M} j}^{(i)}$} \\
\hline & 1 & Состояние & 3 \\
\hline$u_{\mathrm{M} 1}^{(i)}$ & 0 & $-\left(u-u_{\mathrm{H} 2}\right) \frac{w_{1}}{w_{2}+w_{3}}$ & $-\left(u-u_{\mathrm{H} 3}\right) \frac{w_{1}+w_{2}}{w_{3}}$ \\
\hline$u_{\mathrm{M} 2}^{(i)}$ & $\left(u-u_{\mathrm{H} 1}\right) \frac{w_{1}}{w_{1}+w_{2}+w_{3}}$ & 0 & $-\left(u-u_{\mathrm{H} 3}\right) \frac{w_{2}}{w_{3}}$ \\
\hline$u_{\mathrm{M} 3}^{(i)}$ & $\left(u-u_{\mathrm{H} 1}\right) \frac{w_{1}+w_{2}}{w_{1}+w_{2}+w_{3}}$ & $\left(u-u_{\mathrm{H} 2}\right) \frac{w_{2}}{w_{2}+w_{3}}$ & 0
\end{tabular}

В силу отсутствия постоянной составляющей в напряжении магнитных ключей верно уравнение

$$
\int_{\alpha_{1}}^{\alpha_{1}+\pi} u_{\mathrm{M} j}^{(i)} d \Theta=\int_{\alpha_{1}}^{\alpha_{1}+\pi}\left(u_{\mathrm{M} j}^{(1)}+u_{\mathrm{M} j}^{(2)}+u_{\mathrm{M} j}^{(3)}\right) d \Theta=0,
$$

где $j=1,2,3$ и верхние индексы означают состояния схемы.

Поскольку в полупериоде $\alpha_{1} \leqslant \Theta \leqslant \alpha_{1}+\pi$ напряжение $u_{w}=u-u_{\mathrm{H} j}$ изменяет полярность, и отношения (3) и уравнение (5) удовлетворяются, возможен только следующий цикл замыкания (насыщения сердечника) магнитных ключей: $\mathrm{M1} \rightarrow \mathrm{M} 2 \rightarrow \mathrm{M} 3 \rightarrow \mathrm{M} 2 \rightarrow \mathrm{M} 1$. 


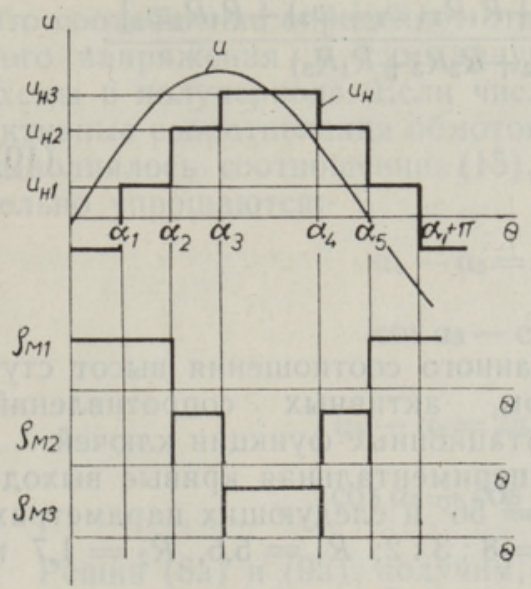

Рис. 3.
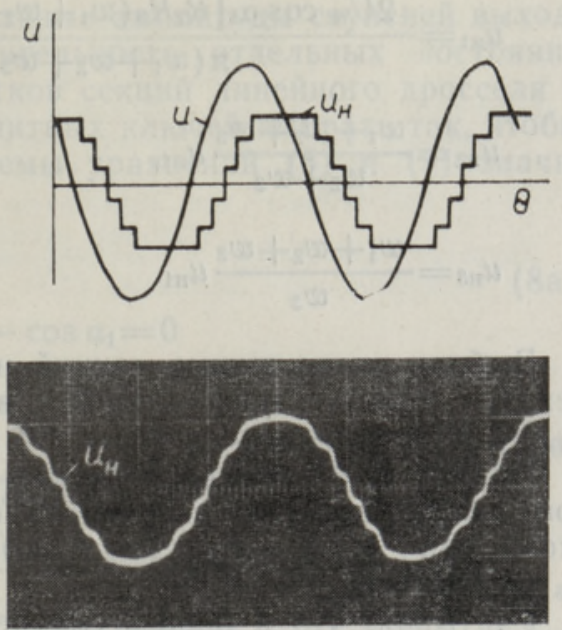

Рис. 4.

Исходя из уравнения (5), найдем соотношения между углами замыкания магнитных ключей $M_{j}$ :

$$
\begin{gathered}
\cos \alpha_{5}-\cos \alpha_{2}-\left(\pi+\alpha_{2}-\alpha_{5}\right) u_{\mathrm{H} 1}^{*}+2 \cos \alpha_{1}=0, \\
\cos \alpha_{3}-\cos \alpha_{4}-\left(\alpha_{4}-\alpha_{3}\right) u_{\mathrm{H} 3}^{*}=0,
\end{gathered}
$$

где $u_{\mathrm{H}}^{*}=u_{\mathrm{H} j} / U_{m}$ - относительная амплитуда ступеней выходного напряжения (см. рис. 3, где $\varrho_{\mathrm{m} j}-$ коммутационные функции ключей).

Далее, используя выражения (3) и (4), получим для вычисления этих углов системы уравнений:

$$
\begin{gathered}
\alpha_{4}-\alpha_{3}=\frac{\pi R_{1} R_{2} w_{3}}{R_{2} R_{3}\left(w_{1}+w_{2}+w_{3}\right)+R_{1} R_{3}\left(w_{2}+w_{3}\right)+R_{1} R_{2} w_{3}}, \\
\cos \alpha_{3}-\cos \alpha_{4}-\frac{2 R_{1} R_{2} \cos \alpha_{1}}{R_{1} R_{2}+R_{1} R_{3}+R_{2} R_{3}}=0
\end{gathered}
$$

И

$$
\begin{gathered}
\alpha_{5}-\alpha_{2}=\frac{\pi R_{1}\left[R_{3}\left(w_{2}+w_{3}\right)+R_{2} w_{3}\right]}{R_{2} R_{3}\left(w_{1}+w_{2}+w_{3}\right)+R_{1} R_{3}\left(w_{2}+w_{3}\right)+R_{1} R_{2} w_{3}}, \\
\cos \alpha_{5}-\cos \alpha_{2}+\frac{2 R_{1}\left(R_{2}+R_{3}\right) \cos \alpha_{1}}{R_{1} R_{2}+R_{2} R_{3}+R_{1} R_{3}}=0 .
\end{gathered}
$$

Учитывая, что среднее значение выходного напряжения регулятора равно $\left[{ }^{3}\right]$

$$
U_{\mathrm{H} \mathrm{cp}}=\frac{1}{\pi} \int_{\alpha_{1}}^{\alpha_{1}+\pi} u_{\mathrm{H} j} d \Theta=\frac{2}{\pi} U_{m} \cos \alpha_{1},
$$

из системы уравнений (8) и (9) получим формулы для вычисления амплитуд ступеней выходного напряжения: 


$$
\begin{aligned}
& u_{\mathrm{H} 1}=\frac{2 U_{m} \cos \alpha_{1}\left[R_{2} R_{3}\left(w_{1}+w_{2}+w_{3}\right)+R_{1} R_{3}\left(w_{2}+w_{3}\right)+R_{1} R_{2} w_{3}\right]}{\pi\left(w_{1}+w_{2}+w_{3}\right)\left(R_{1} R_{2}+R_{2} R_{3}+R_{1} R_{3}\right)}, \\
& u_{\mathrm{H} 2}=\frac{w_{1}+w_{2}+w_{3}}{w_{2}+w_{3}} u_{\mathrm{H} 1}, \\
& u_{\mathrm{H} 3}=\frac{w_{1}+w_{2}+w_{3}}{w_{3}} u_{\mathrm{H} 1} .
\end{aligned}
$$

Выбрав число витков секций из заданного соотношения высот ступеней, можно подходящим выбором активных сопротивлений $R_{1}, R_{2}, R_{3}$ определить из (8) и (9) коммутационные функции ключей.

На рис. 4 показаны расчетная и экспериментальная кривые выходного тока $i_{\mathrm{H}}$ при угле регулирования $\alpha_{1}=56^{\circ}$ и следующих параметрах схемы: $\left(w_{1}+w_{2}+w_{3}\right):\left(w_{2}+w_{3}\right): w_{3}=8: 3: 2 ; R_{1}=5,5, R_{2}=1,7$ и $R_{3}=0,45$ OM.

Учитывая, что в общем случае интервал открытого состояния магнитных ключей $M 1$ и $M 2$ за полупериод $\alpha_{1} \leqslant \Theta \leqslant \alpha_{1}+\pi$ неделим на два равных этапа, т. е. $\alpha_{2}-\alpha_{1} \neq \alpha_{1}+\pi-\alpha_{5}$ и $\alpha_{3}-\alpha_{2} \neq \alpha_{5}-\alpha_{4}$, приведем обобщенные формулы для вычисления коэффициента искажения выходного напряжения

$$
k_{\mathrm{H}}=\frac{U_{\mathrm{H}(1)}}{U_{\mathrm{H}}},
$$

где $U_{\text {н }}$ и $U_{\text {н(1) }}$ - действующие значения выходного напряжения и его первой гармоники, найденные по формулам

$$
\begin{aligned}
& U_{\mathrm{H}}=\sqrt{\frac{1}{\pi}\left[u_{\mathrm{H} 1}^{2}\left(\pi+\alpha_{2}-\alpha_{5}\right)+u_{\mathrm{H} 2}^{2}\left(\alpha_{3}+\alpha_{5}-\alpha_{2}-\alpha_{4}\right)+u_{\mathrm{H} 3}^{2}\left(\alpha_{4}-\alpha_{3}\right)\right]} \\
& U_{\mathrm{H}(1)}=\sqrt{\frac{a_{1}^{2}+b_{1}^{2}}{2}}
\end{aligned}
$$

Амплитуда косинусоидального члена первой гармоники:

$$
\begin{gathered}
a_{1}=\frac{2}{\pi} \int_{\alpha_{1}}^{\alpha_{1}+\pi} u_{\mathrm{H} j} \cos \Theta d \Theta=\frac{2}{\pi}\left\{\left(u_{\mathrm{H} 2}-u_{\mathrm{H} 1}\right)\left[\sin \left(\alpha_{5}-\alpha_{1}\right)-\sin \left(\alpha_{2}-\alpha_{1}\right)\right]+\right. \\
\left.-1-\left(u_{\mathrm{H} 3}-u_{\mathrm{H} 2}\right)\left[\sin \left(\alpha_{4}-\alpha_{1}\right)-\sin \left(\alpha_{3}-\alpha_{1}\right)\right]\right\} .
\end{gathered}
$$

Амплитуда синусоидального члена первой гармоники:

$$
\begin{aligned}
& b_{1}=\frac{2}{\pi} \int_{\alpha_{1}}^{\alpha_{1}+\pi} u_{\mathrm{H} j} \sin \Theta d \Theta=\frac{2}{\pi}\left\{2 u_{\mathrm{H} 1}+\left(u_{\mathrm{H} 2}-u_{\mathrm{H} 1}\right)\left[\cos \left(\alpha_{2}-\alpha_{1}\right)-\right.\right. \\
& \left.\left.-\cos \left(\alpha_{5}-\alpha_{1}\right)\right]+\left(u_{\mathrm{H} 3}-u_{\mathrm{H} 2}\right)\left[\cos \left(\alpha_{3}-\alpha_{1}\right)-\cos \left(\alpha_{4}-\alpha_{1}\right)\right]\right\} .
\end{aligned}
$$

Известно, что в трехступенчатой форме выходного напряжения (тока) отсутствуют все высшие гармоники ниже 11-й, если действительно соотношение $\left[{ }^{1}\right]$

$$
I_{01}: I_{02}: I_{03}=u_{\mathrm{H} 1}: u_{\mathrm{H} 2}: u_{\mathrm{H} 3}=0,2610: 0,7135: 0,9745 .
$$


Это соотношение определяет относительные амплитуды ступеней выходного напряжения и суммарную длительность отдельных состояний схемы в полупериоде. Если число витков секций линейного дросселя и активные сопротивления обмоток магнитных ключей выбрать так, чтобы выполнялось соотношение (15), системы уравнений (8) и (9) значительно упрощаются:

$$
\begin{aligned}
& \alpha_{4}-\alpha_{3}=\pi / 3 \\
& \cos \alpha_{3}-\cos \alpha_{4}-\cos \alpha_{1}=0
\end{aligned}
$$

и

$$
\begin{aligned}
& \alpha_{5}-\alpha_{2}=2 \pi / 3 \\
& \cos \alpha_{5}-\cos \alpha_{2}+1,7322 \cos \alpha_{1}=0
\end{aligned}
$$

Решив (8а) и (9a), получим, что

$$
\alpha_{3}-\alpha_{2}=\pi / 6 \quad \text { и } \quad \alpha_{2}=\alpha_{1}+\pi / 6 .
$$

Из вышеизложенного вытекает, что при выполнении соотношения (15) форма выходного напряжения (тока) не зависит от угла управления мостового регулятора. При этом в нем устраняются все высшие гармоники ниже 11 -й, и в диапазоне $\alpha_{\Pi} \leqslant \alpha_{1} \leqslant \pi / 2$ коэффициент искажения $k_{\mathrm{H}}=0,989$.

Если в качестве переключателей секций линейного дросселя формирователя трехступенчатого тока использовать тиристоры и этими тиристорами управлять магнитными ключами, все приведенные здесь соотношения для вычисления углов замыкания ключей и формы выходного тока (напряжения) сохраняют силу, изменяется лишь определение эквивалентных сопротивлений. Хотя в статье рассмотрен случай с 3 ключами, процесс управления в индуктивно-ключевой цепи формирования многоступенчатого тока одинаков при любом числе ступеней.

\section{Выводы}

1. Применение магнитных ключей в качестве переключателей числа витков линейного дросселя в индуктивно-ключевой цепи формирования многоступенчатого тока позволяет существенно упростить схему управления регуляторов переменного напряжения.

2. При заданном отношении витков секций линейного дросселя форма выходного тока индуктивно-ключевого формирователя определяется активными сопротивлениями обмоток магнитных элементов схемы и углом управления мостового регулятора.

3. При определенном выборе отношений витков секций линейного дросселя и отношений активных сопротивлений обмоток форма выходного тока не зависит от угла управления мостового регулятора. При этом в выходном токе устраняются самые низкие из высших гармоник.

\section{Л И ТЕ Р А Т У Р А}

1. Т онкаль С. С. К гармоническому анализу ступенчатой функции, аппроксимирующей синусоидальное напряжение. - В сб.: Проблемы технической электродинамики, вып. 24 . Киев, 1970 , с. $37-42$.

2. Тонкаль В. Е., Липков ский К. А., Мельничук Л. П. Способы улучшения качества выходного напряжения автономных инверторов. Препринт-49 ИЭД АН УССР. Киев, 1972. 
3. О я в е эр М., С а р в В. Анализ преобразовательных процессов в индуктивно-ключевой цепи непрерывного амплитудного регулирования переменного напряжения. - Изв. АН ЭССР, Физ. Матем., 1977, т. 26, № 3, с. 297-303.

4. О я в е эр М., С а р в В. Процессы преобразования в тиристорно-индуктивной цепи непрерывного амплитудного регулирования переменного напряжения при естественной коммутации. - Изв. АН ЭССР, Физ. Матем., 1978, т. 27, № 3, c. $342-348$.

Ннститут термофизики и электрофизики Академии наук Эстонской ССР

Поступила в редакцию 20/II 1978

J. SOOJARV, T. LAUSMAA

\section{JUHTIMISPROTSESSID MITMEASTMELISE REGULEERITAVA VAHELDUVVOOLU FORMEERIMISE INDUKTIIVLULITUSAHELAS}

Artiklis on kirjeldatud juhtimisprotsesse vahelduvpingeregulaatori induktiivlülitusahelas siinuselähedase mitmeastmelise vahelduvvoolu formeerimisel ja esitatud seosed väljundpinge kuju arvutamiseks, lähtudes ahela parameetritest.

\section{J. SOOJARV, T. LAUSMAA}

\section{CONTROL PROCESSES IN THE INDUCTIVE SWITCHING CIRCUIT FOR MULTI- STEP ADJUSTABLE ALTERNATING CURRENT FORMATION}

Control processes in the inductive switching circuit for adjustable alternating current formation having a multistep waveform close to sinusoidal is described. It is shown that the use of magnetic switches for varying the turn number of the linear reactor in the inductive switching circuit simplifies considerably the regulator circuit. If the turn ratio of the linear reactor winding sections is properly adjusted, the output current waveform is mainly determined by the active winding resistance of magnetic elements in the circuit. With an appropriate choice of the section turn ratio of the linear reactor, the output current waveform does not depend on the regulation angle of the bridge regulator. The necessary formulae for calculating the output voltage waveform as a function of the parameters of the inductive switching circuit are given. 